\title{
In Vivo Measurement of Transverse Relaxation Time in the Mouse Brain at $17.6 \mathrm{~T}$
}

\author{
Firat Kara, ${ }^{1}$ Fu Chen, ${ }^{1}$ Itamar Ronen, ${ }^{2}$ Huub J. M. de Groot, ${ }^{1}$ Jörg Matysik, ${ }^{1}$ and \\ A Alia ${ }^{1,2 *}$
}

Purpose: To establish regional $T_{1}$ and $T_{2}$ values of the healthy mouse brain at ultra-high magnetic field strength of $17.6 \mathrm{~T}$ and to follow regional brain $T_{1}$ and $T_{2}$ changes with age.

Methods: In vivo $T_{1}$ and $T_{2}$ values in the C57BL/6J mouse brain were followed with age using multislice-multiecho sequence and multiple spin echo saturation recovery with variable repetition time sequence, respectively, at 9.4 and $17.6 \mathrm{~T}$. Gadolinium-tetra-azacyclo-dodecane-tetra-acetic acid phantoms were used to validate in vivo $T_{2}$ measurements. Student's $t$-test was used to compare mean relaxation values.

Results: A field-dependent decrease in $T_{2}$ is shown and validated with phantom measurements. $T_{2}$ values at $17.6 \mathrm{~T}$ typically increased with age in multiple brain regions except in the hypothalamus and the caudate-putamen, where a slight decrease was observed. Furthermore, $T_{1}$ values in various brain regions of young and old mice are presented at 17.6 T. A large gain in signal-to-noise ratio was observed at 17.6 T.

Conclusions: This study establishes for the first time the normative $T_{1}$ and $T_{2}$ values at 17.6 T over different mouse brain regions with age. The estimates of in vivo $T_{1}$ and $T_{2}$ will be useful to optimize pulse sequences for optimal image contrast at 17.6 T and will serve as baseline values against which disease-related relaxation changes can be assessed in mice. Magn Reson Med 70:985-993, 2013. @ 2012 Wiley Periodicals, Inc.

Key words: $T_{2}$ relaxation time; ultra-high field $17.6 \mathrm{~T}$; mouse brain; age-related $T_{2}$ and $T_{1}$ changes

\section{INTRODUCTION}

Transverse $\left(T_{2}\right)$ and longitudinal $\left(T_{1}\right)$ relaxation times play an important role in optimizing magnetic resonance imaging (MRI) parameters [e.g., contrast level, signal-to-noise ratio (SNR)] and provide a quantitative indicator of tissue changes induced by various pathological conditions $(1,2)$. The changes in $T_{2}$ values of the brain tissue are widely used as a surrogate marker in the evaluation of various

${ }^{1}$ Solid State NMR, Leiden Institute of Chemistry, Gorlaeus Laboratoria, Leiden, The Netherlands.

${ }^{2}$ Department of Radiology, Leiden University Medical Center, Leiden, The Netherlands.

Additional Supporting Information may be found in the online version of this article.

Grant sponsors: Internationale Stichting Alzheimer Onderzoek (ISAO) and Centre for Medical Systems Biology (CMSB).

${ }^{*}$ Correspondence to: A Alia, Ph.D., Leiden Institute of Chemistry, Gorlaeus Laboratoria, Einsteinweg 55, P.O. Box 9502, Leiden 2300 RA,

The Netherlands. E-mail: a.alia@chem.leidenuniv.nl

Received 24 August 2012; revised 26 September 2012; accepted 27 September 2012.

DOI 10.1002/mrm.24533

Published online 14 November 2012 in Wiley Online Library (wileyonlinelibrary.com)

(c) 2012 Wiley Periodicals, Inc. brain disorders $(3,4)$. The in vivo $T_{2}$ of brain tissue is related to the local water environment and the free-tobound water ratio, which may change in response to cellular and axonal loss or membrane breakdown (5-8). For example, the process of breakdown of myelin in certain types of neurodegenerative disorders, such as multiple sclerosis and Alzheimer's disease, leads to alterations in the local water environment that increase the ratio of the free-to-bound water in tissue and thus increases $T_{2}$ and $T_{1}$ of white matter $(5,9,10)$. The $T_{2}$ and $T_{1}$ also change in brain regions where brain iron is deposited in the form of ferritin and hemosiderin (11). In addition, both $T_{1}$ and $T_{2}$ values provide quantitative information about tissue changes in the healthy brain during aging $(12,13)$.

Mice are routinely used as models for studying human brain disorders. Due to the small size of the mouse brain, the present trend in in vivo mouse brain MRI is to move toward ultra-high magnetic field $(\geq 7 \mathrm{~T})$ for improved SNR, higher resolution, sensitivity, and specificity (14-18). Thus, ultra-high magnetic field systems, such as $17.6 \mathrm{~T}$, can serve as a powerful tool to detect more subtle abnormalities in anatomical and functional characteristics of emerging mouse models of neurodegenerative diseases (19). Although the values for $T_{2}$ relaxation time in different parts of the mouse brain have been published in different field strengths $(2,12,20-23)$, there is no systematic assessment of the regional $T_{2}$ values for the healthy mouse brain at 17.6 T. The values of $T_{1}$ relaxation time of various mouse brain regions have been reported at $17.6 \mathrm{~T}$ in young mice (18). However, age-dependent changes in $T_{1}$ have not been analyzed at this magnetic field strength. The C57BL/6J mouse is one of the most widely used inbred strains and the first to have its genome sequenced (24). It is generally used as a background strain for the generation of transgenic models of various brain diseases such as Alzheimer's disease (19). In this study, we aimed to establish the values of regional $T_{2}$ relaxation time for healthy C57BL/6J mouse brain in vivo at $17.6 \mathrm{~T}$ and to determine magnetic field dependent changes in $T_{2}$. Phantom solutions were used to validate changes in $T_{2}$ at different field strengths and to explore the validity of multislice-multiecho (MSME) sequence to measure $T_{2}$. In addition, we assessed age-related regional $T_{2}$ and $T_{1}$ changes in the mouse brain at $17.6 \mathrm{~T}$. These estimates of in vivo $T_{2}$ and $T_{1}$ relaxation of the mouse brain will be useful to optimize MRI sequences for optimal image contrast and sensitivity in the mouse brain at 17.6 T. In addition, knowledge of age-related $T_{2}$ and $T_{1}$ changes in the healthy mouse brain is mandatory, if disease-related deviations in $T_{2}$ and $T_{1}$ have to be studied in longitudinal studies. 


\section{METHODS}

\section{Phantoms}

Phantom solution containing different concentrations of Gadolinium-tetra-azacyclo-dodecane-tetra-acetic acid (Gd-DOTA) (Dotarem; Guerbet Nederland BV, Gorinchem, The Netherlands) was prepared by diluting a stock solution of Gd-DOTA (0.5 M) in phosphate buffer saline ( $\mathrm{pH}$ 7.50). Dilution factors used for $T_{2}$ measurements were 1/50 (i.e., $10 \mathrm{mmol}$ Gd-DOTA/L), 1/100, 1/ $200,1 / 400,1 / 800)$. The same phantoms were used for both MRI and nuclear magnetic resonance (NMR) experiments. For the SNR analysis, the phantom solution consisting of $20 \% \mathrm{H}_{2} \mathrm{O}, 80 \% \mathrm{D}_{2} \mathrm{O}$, and $1 \mathrm{~g} \mathrm{~L}^{-1}$ copper sulfate $\left(\mathrm{CuSO}_{4}\right)$ was prepared.

\section{High-Resolution NMR}

High-resolution NMR experiments were performed at 2.35, 9.4, and 17.6 $\mathrm{T}$ magnets using a broadband 5-mm solution-state NMR probe. Radiation damping was minimized using a restricted sample volume (capillary NMR tubes) in low-Q probes. The pulse sequence used for $T_{2}$ measurements was based on the Carr-Purcell-MeiboomGill (CPMG) scheme. The pulse lengths of 90 and $180^{\circ}$ at all fields were 9.6 and $19.2 \mu \mathrm{s}$, respectively. A variable list of 16 duration times between 90 and $180^{\circ}$ pulse was adopted. Both the recycle delay and the longest duration time were kept at larger than 10 times of the expected $T_{2}$ of each sample.

\section{Mice}

For all in vivo measurements, female C57BL/6J mice were used. All the animal experiments were approved by the Institutional Animal Care and Animal Use Committee of the University of Leiden in accordance with the NIH Guide for the Care and Use of Laboratory Animals.

\section{MRI}

All MRI measurements were performed using $400 \mathrm{MHz}$ (9.4 T) and $750 \mathrm{MHz}$ (17.6 T) vertical 89-mm bore magnets equipped with a $1 \mathrm{~T} \mathrm{~m}^{-1}$ actively shielded imaging gradient insert (Bruker, Germany). A birdcage radiofrequency (RF) coil with an inner diameter of $2 \mathrm{~cm}$ was used for excitation and detection. The system was interfaced to a Linux PC running Topspin 2.0 and ParaVision 5.0 software (Bruker Biospin GmbH, Germany).

All in vivo MRI studies were conducted as previously described (25). Before imaging, the mice were initially anesthetized with $2 \%$ isoflurane (Forane, Abott, UK), in air $\left(0.3 \mathrm{~L} \mathrm{~min}^{-1}\right)$ and oxygen $\left(0.3 \mathrm{~L} \mathrm{~min}^{-1}\right)$. During scanning, the level of anesthetic was maintained between 1 and $1.5 \%$ to keep the breathing of the animal at a constant rate of $\sim 50$ breaths $\mathrm{min}^{-1}$. Animals were placed in the 20-mm birdcage RF coil with a special mouse head mask, which was used to administer the anesthetic gas during the MR experiments. A respiration sensor, connected to a respiration unit, was placed on the abdomen to monitor respiration rate. The respiration unit was connected to a computer having Bio-SAM respiration moni- toring software (Bruker Biospin, Germany). During MRI imaging, the respiration rate of the mouse was constantly monitored, and the body temperature of the mouse was kept constant by pumping warm water $\left(30 \pm 1^{\circ} \mathrm{C}\right)$ through the gradient system. The rectal body temperature of the mouse during scanning was measured to be $30 \pm$ $1^{\circ} \mathrm{C} . T_{2}$ measurements in phantom solutions were also conducted at $30 \pm 1^{\circ} \mathrm{C}$ and were repeated at least four times.

$T_{2}$ values were measured with the MSME sequence that is based on the CPMG sequence, where transverse magnetization of a $90^{\circ}$ pulse is refocused by a train of $180^{\circ}$ pulses generating a series of echoes $(26,27)$. The following imaging parameters were used for the phantom and in vivo experiments both at 9.4 and $17.6 \mathrm{~T}$ : number of averages $=2$; number of slices $=1$ and/or 10 with a slice thickness of $1.0 \mathrm{~mm}$ and an interslice thickness of $1.5 \mathrm{~mm}$; number of echoes $=16$ with echo spacing $=$ 8.5; a repetition time (TR) $=3 \mathrm{~s}$ with an effective spectral bandwidth $=50 \mathrm{kHz}$; field of view $(\mathrm{FOV})=2.0 \times$ $2.0 \mathrm{~cm}^{2}$; and matrix size $=256 \times 256$; this yields an effective in in-plane resolution of $0.078 \times 0.078 \mathrm{~mm}^{2}$ and a voxel resolution of $6.10 \times 10^{-3} \mathrm{~mm}^{3}$. The total acquisition time for the experiment was $19 \mathrm{~min} 12 \mathrm{~s}$. To study the dependence of $T_{2}$ on the CPMG refocusing interpulse interval $(\tau)$, the $T_{2}$ measurements were performed using the MSME sequence with 16 echo and 4 different refocusing interpulse intervals, namely 5.6, 8.5, 10 , and $18 \mathrm{~ms}$. The last 5 echo of $8.5 \mathrm{~ms}$ acquisition, the last 7 echo of $10 \mathrm{~ms}$ acquisition, and the last 10 echo of $18 \mathrm{~ms}$ acquisition were discarded to provide comparable temporal sampling windows (namely, 5.6-91.04, 8.593.50, 10-90, and 18-90 ms acquisitions, respectively). For the precise localization of regions of interest (ROIs) on the brain regions for $T_{2}$ measurements, a pilot scan of a mouse brain was acquired with multislice rapid acquisition using the relaxation enhancement (RARE) sequence (28), and subsequently ROIs were transferred to $T_{2}$ maps to ensure precise regional placement. The following imaging parameters were used for the RARE sequence: echo time (TE) $=11.67 \mathrm{~ms}$ (at 9.4 T) and 8.5 $\mathrm{ms}$ (at 17.6 T); TR $=1500 \mathrm{~ms}$ (at $9.4 \mathrm{~T}$ ) and $2000 \mathrm{~ms}$ (at $17.6 \mathrm{~T}$ ); number of averages $=1$, rare factor $=6$; number of slices $=10$, with slice thickness $1.0 \mathrm{~mm}$; and interslice thickness $=1.5 \mathrm{~mm}$. To establish the test-retest reliability of $T_{2}$ measurements, the same mice $(n=7)$ were scanned twice with an interval of 4 days, and the phantom solution $(10 \mathrm{mM})$ was scanned twice with an interval of 6 weeks. For the measurement of the SNR in the phantom solution, the mic-MSME sequence (Bruker, Paravision 5) was used. The following imaging parameters were applied both at 9.4 and $17.6 \mathrm{~T}$ : TE $=7 \mathrm{~ms}$, TR $=1000 \mathrm{~ms}$, number of averages $=1, \mathrm{FOV}=6.0 \times 6.0$, matrix $=256 \times 256$, slice thickness $=1 \mathrm{~mm}$, and number of slices $=11$.

$T_{1}$ was measured with a multiple spin echo saturation recovery method with variable TR (RAREVTR). Slice excitation and refocusing were accomplished by hermite pulses, resulting in 90 and $180^{\circ}$ pulse lengths of 1.4 and $0.9 \mathrm{~ms}$, respectively. The following imaging parameters were used at $17.6 \mathrm{~T}$ : TE $=5.5 \mathrm{~ms}$; TR-array $=0.1,0.18$, $0.36,0.6,0.8,1.0,1.5,2.0,4.0,6.0,10.0$, and $15.0 \mathrm{~s}$; 

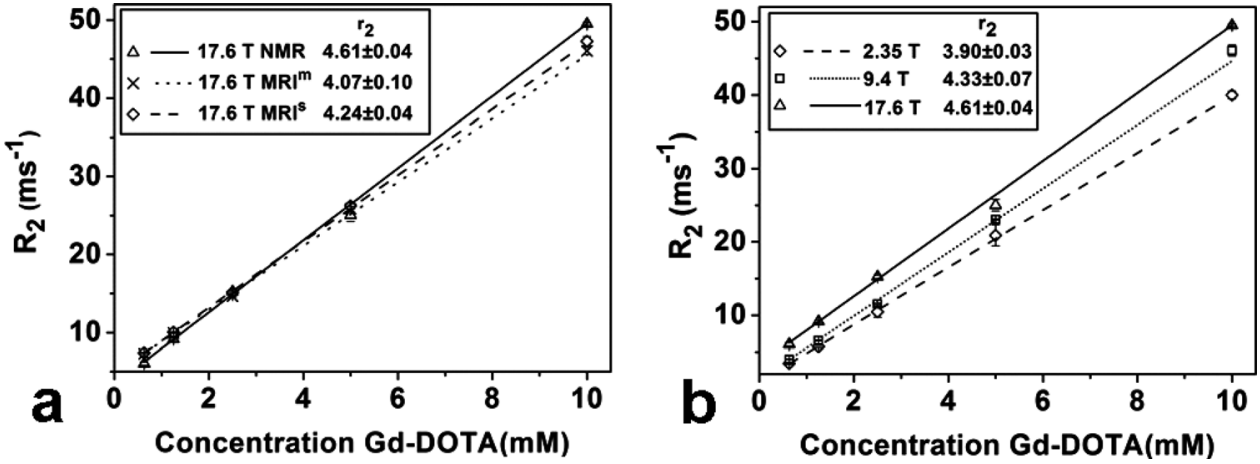

FIG. 1. a: Scatter plots showing the changes in relaxation rates $\left(R_{2}\right)$ as a function of the Gd-DOTA concentration measured using MRI and NMR at 17.6 T. b: Scatter plots showing the changes in $R_{2}$ as a function of magnetic field strength $(2.35$, 9.4, and $17.6 \mathrm{~T})$ measured using NMR. Mean $R_{2}$ in $\mathrm{s}^{-1} \pm$ error bars (SEM: standard error of the mean). The slopes of the weighted linear regression curves are the $T_{2}$ relaxivities $\left(r_{2}\right) \cdot r_{2}$ in $\mathrm{mM}^{-1} \mathrm{~s}^{-1} \pm \mathrm{SEM}$; $\mathrm{MRI}^{\mathrm{m}}$, multislice-multiecho MRI; MRI ${ }^{\mathrm{S}}$, single-slice-multiecho MRI. The correlation coefficient (Pearson's $r$ ) of the linear fitting for all lines is 0.99 .

matrix size $=128 \times 128 ; \mathrm{FOV}=1.7 \times 1.7 \mathrm{~cm}^{2}$; and slice thickness $=0.9 \mathrm{~mm}$. The total acquisition time for the experiment was 33 min $13 \mathrm{~s}$. All images acquired single slices to prevent interslice modulation effects. The slice was positioned as described previously (18). To minimize the contribution of partial volume effect from cerebrospinal fluid, the ROIs were checked carefully on images collected with very thin slices $(0.25 \mathrm{~mm})$ using the same RAREVTR sequence with following parameters: $\mathrm{TR}=6000 \mathrm{~ms}$, TE $=17 \mathrm{~ms}, \mathrm{FOV}=1.5 \times 1.5 \mathrm{~cm}$, matrix size $=256 \times 256$, and number of slices $=10$.

For the comparison of the SNR for in vivo imaging at two different magnetic field strengths (9.4 and 17.6 T), the experiment was performed with the RAREVTR sequence using three different TR $(6,10$, and $15 \mathrm{~s})$ and TE $(5.5,16.5$, and $27.5 \mathrm{~ms}$ ). The following imaging parameters were used: number of averages $=1$; TR-array $=$ 15,000, 10,000, 6000; $\mathrm{TE}=5.5,16.5,27.4 \mathrm{~ms} ; \mathrm{FOV}=1.7$ $\times 1.7 \mathrm{~cm}^{2}$; matrix size $=128 \times 128$; slice thickness $=0.6$ $\mathrm{mm}$; and effective spectral bandwidth $=71,428 \mathrm{kHz}$. The same mouse was used for SNR measurements at 9.4 and 17.6 T.

\section{Data Processing}

Estimation of $T_{2}$ and $T_{1}$

To calculate $T_{2}$, ROIs were drawn on the images using an image sequence analysis tool package (Paravision 5, Bruker), which uses a fit function $[y=A+C * \exp (-t /$ $T_{2}$ )], where $A=$ absolute bias, $C=$ signal intensity, and $T_{2}=$ transverse relaxation time. The $T_{1}$ values were determined by image sequence analysis using a fit function: $M(t)=A+M_{0} *\left(1-\exp \left(t / T_{1}\right)\right.$, where $M_{0}$ is the equilibrium magnetization. ROIs were manually defined for the hippocampus (HC), cortex (CX), thalamus (TH), hypothalamus (HT), corpus callosum (CC), caudate-putamen (Cpu), olfactory bulb (OB) and its glomerular layer (GL), and muscle (M), using the "Allen Brain Atlas" with the brain explorer program (http://mouse.brain-map.org) as the reference atlas. For all animals, the $T_{2}$ and $T_{1}$ were the mean of the ROIs drawn on the right and left sides of the brain, except for the CC region. For phantoms, a cylinder was drawn on the inside of the axial fig- ures of the tubes. The transverse relaxation rate $\left(R_{2}\right)$ was obtained from the equation: $R_{2}=1 / T_{2}\left(\mathrm{~s}^{-1}\right)$. Relaxivity of the contrast agent is defined as the efficiency by which an MRI contrast agent can accelerate the proton relaxation rate in a homogeneous medium (29). Relaxivity $\left(r_{2}\right)$ was calculated as the slope of the linear regression line of a plot of $R_{2}$ vs. concentration of MRI contrast agent (30).

\section{Estimation of SNR}

The SNR in the images is measured as the average signal intensity over the ROI divided by the standard deviation of the noise.

\section{Statistics}

A paired and/or two-tailed Student's $t$-test was used to compare mean values. Statistical significance was assigned for values of $P<0.05$. The reliability of the measurements was assessed by computing the intraclass correlation coefficient $\left(\mathrm{ICC}_{2,1}\right)$ using a two-way random effects analysis of variance (ANOVA) model and the absolute agreement definition $(31,32)$. The associations between test and retest data were analyzed by the Pearson's correlation coefficient $(r)$ at a 0.05 significance level. An ICC close to 1 indicates excellent reliability. The Pearson's $r$ close to 1 indicates excellent association (31).

\section{RESULTS}

\section{Phantom Studies}

Figure 1a depicts the quantitative relaxivity $\left(r_{2}\right)$ of GdDOTA phantom solutions at 17.6 T using MRI and highresolution NMR methods. The MSME and the singleslice-multiecho MRI methods showed 11 and 8\% lower $r_{2}$ values, respectively, relative to the value acquired with the NMR method. Figure $1 \mathrm{~b}$ shows the $r_{2}$ of GdDOTA phantom solutions determined at 2.35, 9.4, and 17.6 $\mathrm{T}$ using the high-resolution NMR method. As is clear from this figure, plots of the transverse relaxation rate, $R_{2}\left(1 / T_{2}\right)$, vs. the Gd-DOTA concentration $(\mathrm{mM})$ reveal a linear dependence of the $R_{2}$ on the 

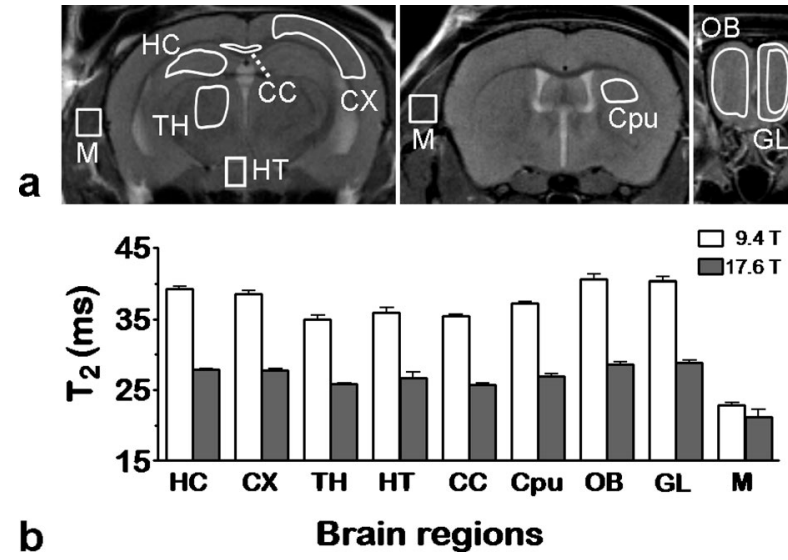

b

FIG. 2. a: MR coronal slices of a mouse brain, showing relevant ROIs, acquired with the RARE sequence at $17.6 \mathrm{~T}$. b: In vivo $T_{2}$ relaxation times of different mouse brain regions acquired at 9.4 and 17.6 T. The analyzed brain structures include the $\mathrm{HC}, \mathrm{CX}, \mathrm{TH}$, $\mathrm{HT}, \mathrm{CC}, \mathrm{Cpu}, \mathrm{OB}, \mathrm{GL}$ of OB; muscle (M, internal reference). Values are expressed as mean $T_{2}$ in ms \pm SEM (error bars). Twotailed Student's $t$-test, $P<0.05$ in all regions; $n=5$.

concentration. In addition, the $r_{2}$ values increased with increasing field strength. The value of $r_{2}$ at $17.6 \mathrm{~T}$ is $\sim 18 \%$ and at $9.4 \mathrm{~T}$ is $\sim 11 \%$ higher relative to the value at $2.35 \mathrm{~T}$. These results clearly show that $T_{2}$ relaxation times of Gd-DOTA decrease with increasing field strength.

To confirm that the $180^{\circ}$ pulses in the MSME sequence did not introduce spurious magnetization from stimulated echoes or from signals originating from outside the selected slices, $T_{2}$ decay curves from a Gd-DOTA phantom with echo spacings of $8.5,13,15$, and $17 \mathrm{~ms}$ were acquired. An example of a $T_{2}$ decay curve with an 8.5 echo spacing is depicted in Figure 1S-a (Supporting Information). The differences among $T_{2}$ values acquired using the various echo spacings were statistically insignificant. To observe the variation of the $T_{2}$ between the 10 slices used in the multiecho sequence, the $T_{2}$ of the phantom (10 $\mathrm{mM}$ ) was measured for each slice. No statistically significant variation in $T_{2}$ values was observed between the slices $(P>0.05)$ (Fig. 1S-b). To assess the test-retest reliability of the Gd-DOTA phantom, $T_{2}$ data were acquired at 17.6 $\mathrm{T}$ using the MSME sequence at two different time points, separated by an interval of 46 days. The differences in mean $T_{2}$ values between two sessions were statistically insignificant, with $P>0.05$. The ICC and Pearson's $r$ values were measured as 0.92 and 0.97 , respectively, suggesting superior test-rest reliability $(P<0.05$ in all cases) and minimum instrumental variations. The SNR performance at both magnetic field strengths was compared and was found to increase by a factor of 1.5 at $17.6 \mathrm{~T}$ as compared to $9.4 \mathrm{~T}$.

\section{In Vivo Studies}

Figure 2a shows ROIs that were selected on a representative series of RARE images from a normal mouse to acquire $T_{2}$. Figure $2 \mathrm{~b}$ depicts a graphical summary of the in vivo $T_{2}$ relaxation times in the mouse brain, at 9.4 and 17.6 T. A clear decrease in $T_{2}$ values was observed in vari- ous brain regions at 17.6 $\mathrm{T}$ as compared to 9.4 T. However, the $T_{2}$ in the muscle showed a relatively small decrease at $17.6 \mathrm{~T}$ as compared to $9.4 \mathrm{~T}$. At both magnetic field strengths, the $T_{2}$ were the longest for the $\mathrm{OB}$ and the grill regions, whereas the TH and the CC consistently show a shorter $T_{2}$ compared with other brain regions. Table 1 depicts the quantitative summary of the in vivo $T_{2}$ of the mouse brain at 9.4 and $17.6 \mathrm{~T}$. To validate the in vivo $T_{2}$ dataset that was acquired using a multislice mode, the measurement was repeated in the single-slice mode. The $T_{2}$ values measured using multislice mode were slightly higher than those from the single-slice mode (Fig. 2S, Supporting Information); however, the systematic overestimation was less than $3 \mathrm{~ms}$. The SNR of the CX region of the mouse brain at both magnetic field strengths was compared and was found to increase by a factor of 1.6 at $17.6 \mathrm{~T}$ as compared to $9.4 \mathrm{~T}$. The dependence of CX and CC $T_{2}$ on the CPMG refocusing interpulse interval $(\tau)$ was investigated at 17.6 T (Fig. 3S, Supporting Information). No statistically significant effect of interpulse interval in the range of interest (between 5.6 and $18 \mathrm{~ms}$ ) was observed on $T_{2}$ of CX and CC.

Table 2 shows test-retest reliability results of in vivo $T_{2}$ measurements in multiple brain regions. The ICCs were statistically significant $(P<0.05)$ for all regions. The test-retest reliability was very good for CX, TH, CC, $\mathrm{Cpu}$, as well as muscle tissue, with the ICC ranging between 0.84 and 0.92 . For HC and HT, ICC was 0.64 and 0.53 , respectively. Table 2 also presents Pearson's $r$, which showed superior association for all regions between two sessions. For all the brain regions, Pearson's $r$ was statistically significant $(P<0.05)$. However, Pearson's $r$ for HT regions was somewhat less than for the other regions $(P=0.062)$. The systematic error was checked by a paired $t$-test, and results are shown in Table 2.

Figure 3 summarizes the age-related changes of the $T_{2}$ for the mouse brain at 17.6 T. The in vivo $T_{2}$ increases with age for multiple brain regions except the HT and the $\mathrm{Cpu}$, where a slight decrease in $T_{2}$ was observed. A significant increase in $T_{2}$ was observed in the CX region in 13.6- and 15.1-month-old mice as compared to 3.6month-old mice (Table 3).

Table 4 depicts the $T_{1}$ values in multiple mouse brain regions in young ( 3 months old) and old (23 months old) mice at $17.6 \mathrm{~T}$. No significant changes in $T_{1}$ were observed between young and old mice in various brain regions except slightly shorter $T_{1}$ in the CC and the Cpu, and slightly longer $T_{1}$ in $\mathrm{TH}$ regions were observed in old mice as compared to young mice.

\section{DISCUSSION}

\section{Phantom Studies}

The $r_{2}$ acquired with the MSME sequence was in very close agreement with the values obtained using highresolution NMR method and the $r_{2}$ derived from the data collected with the single-slice-multiecho sequence. The results validate the accuracy of the $T_{2}$ extracted from the data acquired with the MSME sequence at $17.6 \mathrm{~T}$. In addition, phantom experiments showed that $r_{2}$ decreases with increasing magnetic field strength. The apparent 
decrease of the $T_{2}$ with increasing field strength is incompatible with straightforward Bloembergen-PurcellPound model dipolar relaxation processes that would lead to a $T_{2}$ that is independent of the field strength (14,33). A decrease of the $T_{2}$ with increasing field strength can be attributed to increasing microscopic susceptibility gradients, causing irreversible dephasing due to diffusion across those gradients (34-36). The MSME method used in this study to acquire $T_{2}$ data is timeeffective, because the sequence allows for imaging several slices in a single scan $(37,38)$. Previous studies, however, have reported that many factors, such as spurious echoes, can influence the ability to obtain accurate $T_{2}$ data using the MSME method (37-42). Moreover, there are additional concerns regarding the effect of higher magnetic field strength including: magnetic field inhomogeneity, increased stimulated echo artifacts, and increased power deposition (43). We have demonstrated that the $180^{\circ}$ pulses in the MSME sequence introduce no spurious magnetization contribution from stimulated echoes, because the estimated $T_{2}$ values for the phantom did not change significantly for echo spacings of 8.5, 13, 15 , and $17 \mathrm{~ms}$. Also spatially uniform $T_{2}$ was observed throughout the 10 slices suggesting that the performance of the $180^{\circ}$ refocusing trains was spatially uniform for the MSME sequence (Fig. 1S-b, Supporting Information). Furthermore, excellent test-retest $T_{2}$ reliability was measured by the MSME sequence. These results suggested that there is no evidence of contamination of $T_{2}$ via spurious echoes, and the instrumental variation was negligible at $17.6 \mathrm{~T}$.

A higher SNR was observed for the phantom and the mouse brain at $17.6 \mathrm{~T}$ as compared to $9.4 \mathrm{~T}$. If the relaxation parameters are ignored, the SNR is expected to increase linearly with magnetic field strength (14). The SNR has been reported to be proportional to magnetic field strength $\left(B_{0}\right)$ and the square root of $\left(T_{2} / T_{1}\right)$ in a fully relaxed pulse $\left[\mathrm{SNR} \propto B_{0} \sqrt{ } T_{2} / T_{1}\right]$ (14). The lowerthan-predicted increase in SNR at $17.6 \mathrm{~T}$ could be related with many factors such as changes in relaxation times (shorter $T_{2}$ and longer $T_{1}$ ), increased susceptibility effects, and larger field inhomogeneity $(44,45)$. All these factors can limit the SNR increment at higher fields. In

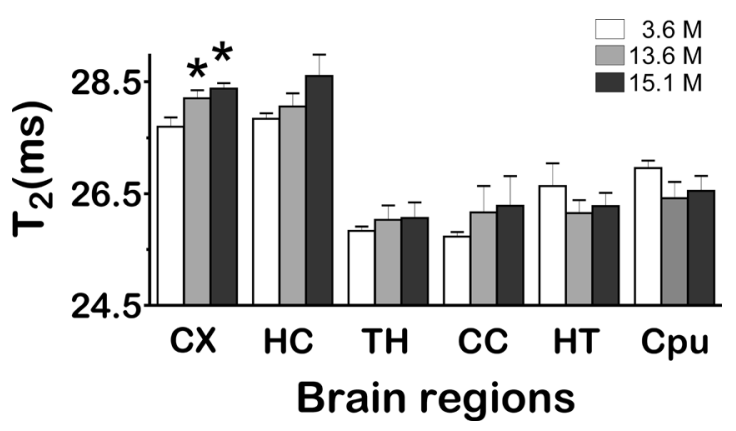

FIG. 3. In vivo $T_{2}$ relaxation times of the mouse brain with age measured at 17.6 T. The analyzed brain structures include the $\mathrm{HC}$, $\mathrm{CX}, \mathrm{TH}, \mathrm{HT}, \mathrm{CC}$, and Cpu. Values are expressed as mean $T_{2}$ in $\mathrm{ms} \pm \mathrm{SEM}$ (error bars); $n=5$. Paired $t$-test, ${ }^{\star} P<0.05$, significant from $T_{2}$ at 3.6 months (M). Same mice were scanned at the age of 3.6, 13.6, and 15.1 months. 
Table 2

Test-Retest Reliability Results of In Vivo $T_{2}$ Relaxation in Various Mouse Brain Regions at $17.6 \mathrm{~T}$

\begin{tabular}{|c|c|c|c|c|c|}
\hline \multirow[b]{2}{*}{ Structure } & \multicolumn{2}{|c|}{ Mean \pm SEM } & \multirow[b]{2}{*}{$\mathrm{ICC}_{2,1}$} & \multirow[b]{2}{*}{$r$} & \multirow[b]{2}{*}{ Paired $t$-test } \\
\hline & Session 1 & Session 2 & & & \\
\hline CX & $28.11 \pm 0.14$ & $28.01 \pm 0.15$ & 0.92 & 0.95 & ns \\
\hline $\mathrm{HC}$ & $28.24 \pm 0.21$ & $27.97 \pm 0.14$ & 0.64 & 0.79 & ns \\
\hline $\mathrm{TH}$ & $26.09 \pm 0.18$ & $25.90 \pm 0.16$ & 0.84 & 0.91 & ns \\
\hline HT & $26.28 \pm 0.18$ & $25.79 \pm 0.30$ & 0.53 & 0.73 & ns \\
\hline $\mathrm{CC}$ & $26.43 \pm 0.44$ & $26.37 \pm 0.30$ & 0.82 & 0.86 & ns \\
\hline Cpu & $26.47 \pm 0.20$ & $26.27 \pm 0.21$ & 0.84 & 0.88 & ns \\
\hline OB & $27.50 \pm 0.27$ & $27.25 \pm 0.24$ & 0.90 & 0.97 & $<0.05$ \\
\hline $\mathrm{GL}$ & $27.56 \pm 0.24$ & $27.22 \pm 0.21$ & 0.83 & 0.96 & $<0.05$ \\
\hline $\mathrm{M}$ & $21.47 \pm 0.22$ & $21.37 \pm 0.21$ & 0.90 & 0.90 & ns \\
\hline
\end{tabular}

$\overline{\mathrm{ICC}}=$ intraclass correlation using a two-way random effects ANOVA (subject by session) and the absolute agreement, $P<0.05$ in all cases for ICC, $r=$ Pearson correlation coefficient, $P<0.05$ in all cases for $r$ except for HT, where $P=0.062, T_{2}$ (ms) means from seven subjects, SEM = standard error of the mean, paired $t$-test = session 1 and session 2 results collected from the same mice were compared. $P>0.05$ in all cases for paired $t$-test except for OB and GL, where $P<0.05$. There is four-day interval between session 1 and session 2 .

addition, it has been reported that experimental conditions such as RF coils, pulse sequences, and preamplifiers also affect the SNR quantification (46).

\section{In Vivo Studies}

In this study, the in vivo $T_{2}$ for several regions of the healthy mouse brain have been presented at $17.6 \mathrm{~T}$. Although the $T_{2}$ in different parts of the transgenic and nontransgenic mouse brain have been published for different field strengths $(2,12,20-23)$, and $T_{2}$ maps of the ischemic mouse brain were provided at $17.6 \mathrm{~T}$, a systematic assessment of the $T_{2}$ for the healthy mouse at $17.6 \mathrm{~T}$ was not yet obtained. Our results at $17.6 \mathrm{~T}$ are well in line with data from healthy rat brain acquired at $16.4 \mathrm{~T}$ (47). In addition, the in vivo $T_{2}$ of the mouse brain determined at 9.4 $\mathrm{T}$ in this study reproduces well with earlier published data $(2,12,20,22)$. In particular, the $T_{2}$ values of the CC, HC, and Cpu are in excellent agreement with earlier report at $9.4 \mathrm{~T}(20)$. Our results also illustrate that $\mathrm{T}_{2}$ decreases in various brain regions with increasing magnetic field strength and are consistent with earlier reports $(14,36,43,48,49)$, although the extent of $T_{2}$ reduction was found to be dependent on the composition of the tissue. The $T_{2}$ of the muscle tissue did not show a strong field dependence, which could be attributed to the lower intracellular molecular mobility of the water in the muscle tissue than for the brain tissue. The decrease of tissue $T_{2}$ with increasing magnetic field strength has been explained in terms of the molecular processes of diffusion and/or chemical exchange of spins between regions with different magnetic field strengths $(34,36,50,51)$. Magnetic field inhomogeneities increase linearly with increasing magnetic field strength and are created by local susceptibility gradients, which can be microscopic (e.g., surrounding blood vessels) as well as macroscopic (e.g., around the sinuses and petrous bone) (34). Diffusion across these magnetic field gradients dephases the transverse magnetization of the water protons, resulting in an overall decrease of the $T_{2}$. The magnitude of these gradients increases with field strength, and therefore, their effect on $T_{2}$ increases with the magnetic field strength (14). This process is referred to as dynamic dephasing, and it depends, among other things, on the refocusing interpulse interval $(\tau)$ defined as half the interval between successive $180^{\circ}$ pulses in a CPMG sequence $(11,36,52)$. It has been shown, in theory and experiments, that $T_{2}$ decreases with increasing $\tau$ $(11,36,52,53)$. Several works have investigated the effect of varying $\tau$ on $T_{2}$ measurements in biological tissues $(11,51,52,54,55)$. In our experiments, to obtain an estimate of the effect that the refocusing interpulse interval has on $T_{2}$, we repeated the $T_{2}$ measurements with four different $\tau$ values, between 5.6 and $18 \mathrm{~ms}$ (Fig. 3S, Supporting Information). No statistically significant dependence of the $T_{2}$ on the interpulse interval was observed. This result suggests that the changes observed in $T_{2}$ values with age depend more on changes in tissue properties in the individual brain structures rather than magnetic field disturbances. In our study, a relatively short refocusing interpulse interval $(8.5 \mathrm{~ms})$ was used. However, even in these conditions, contribution of diffusion and/or chemical exchange on $T_{2}$ is inevitable. In practice, most studies are performed with $\tau$ values no shorter than those used here, and thus, for optimization of contrast and general clinical applications, the $T_{2}$ values

Table 3

In Vivo $T_{2}$ Values of the Mouse Brain with Age at $17.6 \mathrm{~T}$

\begin{tabular}{lccccccc}
\hline Age (months) & $\mathrm{HC}$ & $\mathrm{CX}$ & $\mathrm{TH}$ & $\mathrm{HT}$ & $\mathrm{CC}$ & $\mathrm{Cpu}$ & $\mathrm{M}$ \\
\hline 3.6 & $27.84 \pm 0.10$ & $27.70 \pm 0.16$ & $25.83 \pm 0.08$ & $26.64 \pm 0.41$ & $25.73 \pm 0.08$ & $26.96 \pm 0.13$ & $21.25 \pm 0.44$ \\
13.6 & $28.06 \pm 0.24$ & $28.20 \pm 0.15^{\star}$ & $26.03 \pm 0.26$ & $26.15 \pm 0.23$ & $26.17 \pm 0.48$ & $26.42 \pm 0.29$ & $21.53 \pm 0.31$ \\
15.1 & $28.60 \pm 0.38$ & $28.38 \pm 0.10^{\star}$ & $26.06 \pm 0.28$ & $26.28 \pm 0.23$ & $26.28 \pm 0.53$ & $26.55 \pm 0.27$ & $21.23 \pm 0.16$ \\
\hline
\end{tabular}

Values are expressed as mean relaxation times (ms \pm standard error of the mean), $n=5 . \mathrm{M}=$ muscle.

*Paired two-tailed $t$-test: $P<0.05$ compared to mice at the age of 3.6 months. 
Table 4

In Vivo $T_{1}$ Values of the Mouse Brain at 17.6 T

\begin{tabular}{lcl}
\hline Tissue & Young & Old \\
\hline $\mathrm{HC}$ & $2.14 \pm 0.02$ & $2.16 \pm 0.01$ \\
$\mathrm{CX}$ & $2.02 \pm 0.02$ & $2.07 \pm 0.02$ \\
$\mathrm{TH}$ & $2.05 \pm 0.02$ & $2.17 \pm 0.02^{\star}$ \\
$\mathrm{CC}$ & $1.99 \pm 0.00$ & $1.92 \pm 0.02^{\star}$ \\
$\mathrm{Cpu}$ & $2.01 \pm 0.01$ & $1.96 \pm 0.02^{\star}$ \\
$\mathrm{OB}$ & $1.97 \pm 0.01$ & $1.96 \pm 0.02$ \\
$\mathrm{M}$ & $2.39 \pm 0.03$ & $2.37 \pm 0.01$ \\
\hline
\end{tabular}

Mean $T_{1}$ relaxation times of young ( 3 months) and old (23 months) mice ( $\mathrm{s} \pm$ standard error of the mean), $n=5$. $\mathrm{M}=$ muscle.

*Two-tailed $t$-test: $P<0.05$ compared to young mice.

represented here are expected to be valid and reproducible under similar experimental conditions across platforms.

In our study, age-dependent changes in $T_{2}$ in multiple brain regions, including the $\mathrm{CX}, \mathrm{HC}, \mathrm{TH}$, and $\mathrm{CC}$ were observed at $17.6 \mathrm{~T}$ (Fig. 3 and Table 3 ). In particular, in CX $T_{2}$ relaxation time shows a significant increase with age. These results are in good agreement with a recently published age-dependent $T_{2}$ measurement in human brain at $3 \mathrm{~T}$ (13). $T_{2}$ values of the brain tissue can increase with age in response to cellular and axonal loss or membrane breakdown $(5,56,57)$. These aging processes reduce the number of macromolecules and increase free water content and thus raising the ratio of free-to-bound water protons and as a result contributing to the increase in $T_{2}$ values (13). The lower $T_{2}$ values in the younger mice as compared to the older mice might also be caused by the increased susceptibility effects at $17.6 \mathrm{~T}$. The higher susceptibility effects in the younger mice due to very high ratio of air space to brain tissue may reduce $T_{2}$ values especially at higher fields (17.6 T). Nevertheless, $T_{2}$, acquired in this study using shorter interpulse interval $(\tau=8.5 \mathrm{~ms})$, is in plateau region and seems to be less sensitive to magnetic disturbances induced by local magnetic susceptibility effects. This would also indicate that the changes observed in $T_{2}$ values with age in our study are more dependent on tissue cellularity changes, rather than magnetic disturbances. A few mouse brain regions such as the $\mathrm{HT}$ and the $\mathrm{Cpu}$ show a slight decrease in $T_{2}$ values with age, which could result from increased deposition of paramagnetic substances such as ferritin and hemosiderin $(11,58)$. In human, basal ganglia, e.g., Cpu structures show increased levels of paramagnetic substances with age (13). Falangola et al. (22) did not observe any age-dependency of $T_{2}$ in normal mouse brain at $7 \mathrm{~T}$. Some possible explanations of the differences in the findings can be explained by the ROI definition, use of different imaging parameters, susceptibility effects, and the field strength. Specially, the ROI used in our study covers only the somatosensory and the retrosplenial granular CX regions. However, in the study of Falangola et al., the ROI covers a bigger volume, i.e., the ectorhinal, perirhinal, piriform, somatosensory, and retrosplenial granular CX regions.

It is known that technical challenges such as increased RF field inhomogeneity, increased RF power deposition, and susceptibility effects are prevalent at higher fields
(15), which can cause errors in the measurements of $T_{2}$ $(39,40)$. However, our results show that these factors have negligible affect on the test-retest reliability and accuracy of the phantom and in vivo experiments. Although the MSME sequence provides considerable time savings compared to single-slice-multiecho sequence, cross-talk between slices can occur (59). However, this cross-talk can be substantially decreased by adding slice gaps between adjacent slices (60). In our study, an interslice gap of $150 \%$ of the slice thickness was used that substantially reduced the influence of cross-talk between slices (60). In addition, it has been reported that introducing gaps between neighboring slices also helps in reducing magnetization transfer effects by increasing the resonance offset (59). A slight overestimation of the in vivo $T_{2}$ was observed with the MSME sequence compared to the single-slice sequence at 17.6 T. However, the $T_{2}$ of the mouse brain observed with the MSME sequence were consistently within $3 \mathrm{~ms}$ of the values acquired with the single-slice sequence for all regions (Fig. 2S, Supporting Information).

In clinical evaluations, $T_{2}$ values are often inaccurate in absolute terms due to the systematic errors related to, e.g., the type of curve fitting used in the analysis and the echo train length. In clinical evaluations, however, a good test-retest reliability of $T_{2}$ and shorter scan times are more important than absolute accuracy. The observation that the increase in $T_{2}$ using the MSME sequence as compared to the single-slice-multiecho sequence is relatively small, the absence of obvious interslice variation of $T_{2}$ in multislice imaging, and good test-retest reliability indicated that the MSME protocol can serve to find optimal parameters for $T_{2}$ weighted sequences at $17.6 \mathrm{~T}$. In addition, the MSME sequence can be safely used for evaluation of disease progression in the mouse models of neurodegenerative diseases at 17.6 T.

In addition to $T_{2}$ values, we also measured in vivo $T_{1}$ changes for a variety of brain regions in young (3 months of age) and old (23 months of age) mice at $17.6 \mathrm{~T}$. The $T_{1}$ values obtained in young mice are in excellent agreement with an earlier report at $17.6 \mathrm{~T}$ (18). Comparison of the $T_{1}$ values in multiple brain regions did not show any significant differences in young and old mice. Except, a slightly lower $T_{1}$ was observed in the CC and the Cpu regions in old mice as compared to young mice. These age-dependent $T_{1}$ changes are in agreement with measurements in humans (at $1.5 \mathrm{~T}$ ) and rats (at $16.4 \mathrm{~T}$ ) (47,61-63). In addition, we also observed a slightly higher $T_{1}$ values in $\mathrm{TH}$ region of old mice as compared to young mice. An increase in $\mathrm{TH} T_{1}$ values with age has also been reported in human at $1.5 \mathrm{~T}(63,64)$. The agerelated changes in $T_{1}$ might reflect various physiological processes. For example, an increase in iron content of brain tissue can lead to a decrease in $T_{1}$, whereas a decline in the number of myelinated fibers can cause an increase in $T_{1}(10,11)$.

\section{CONCLUSIONS}

Our results provide a comprehensive and quantitative in vivo $T_{2}$ profile for various mouse brain regions at $17.6 \mathrm{~T}$ and show that the $T_{2}$ decreases substantially at $17.6 \mathrm{~T}$ 
compared with 9.4 T. In addition, age-related $T_{2}$ and $T_{1}$ changes in the mouse brain were observed at $17.6 \mathrm{~T}$, which can provide a useful reference for comparison with disease-related deviations in $T_{2}$ and $T_{1}$ relaxation for future studies.

\section{ACKNOWLEDGMENTS}

The authors thank Kees Erkelens, Karthick Babu Sai Sankar Gupta, and Fons Lefeber for their assistance during various stages of MRI measurements.

\section{REFERENCES}

1. Damadian R. Tumor detection by nuclear magnetic resonance. Science 1971;171:1151-1153.

2. Helpern JA, Lee SP, Falangola MF, et al. MRI assessment of neuropathology in a transgenic mouse model of Alzheimer's disease. Magn Reson Med 2004;51:794-798.

3. Deoni SC. Quantitative relaxometry of the brain. Top Magn Reson Imaging 2010;21:101-113.

4. Ongur D, Prescot AP, Jensen JE, Rouse ED, Cohen BM, Renshaw PF, Olson DP. T2 relaxation time abnormalities in bipolar disorder and schizophrenia. Magn Reson Med 2010;63:1-8.

5. Seewann A, Vrenken H, van der Valk P, Blezer EL, Knol DL, Castelijns JA, Polman CH, Pouwels PJ, Barkhof F, Geurts JJ. Diffusely abnormal white matter in chronic multiple sclerosis: imaging and histopathologic analysis. Arch Neurol 2009;66:601-609.

6. Fullerton GD, Potter JL, Dornbluth NC. NMR relaxation of protons in tissues and other macromolecular water solutions. Magn Reson Imaging 1982;1:209-226.

7. Beaulieu C, Fenrich FR, Allen PS. Multicomponent water proton transverse relaxation and T2-discriminated water diffusion in myelinated and nonmyelinated nerve. Magn Reson Imaging 1998;16: 1201-1210.

8. Jones CK, Whittall KP, MacKay AL. Robust myelin water quantification: averaging vs. spatial filtering. Magn Reson Med 2003;50: 206-209.

9. Bartzokis G. Age-related myelin breakdown: a developmental model of cognitive decline and Alzheimer's disease. Neurobiol Aging 2004; 25:5-18; author reply 49-62.

10. Lacomis D, Osbakken M, Gross G. Spin-lattice relaxation (T1) times of cerebral white matter in multiple sclerosis. Magn Reson Med 1986;3:194-202.

11. Vymazal J, Brooks RA, Baumgarner C, Tran V, Katz D, Bulte JW, Bauminger R, Di Chiro G. The relation between brain iron and NMR relaxation times: an in vitro study. Magn Reson Med 1996;35:56-61.

12. Guilfoyle DN, Dyakin VV, O'Shea J, Pell GS, Helpern JA. Quantitative measurements of proton spin-lattice (T1) and spin-spin (T2) relaxation times in the mouse brain at 7.0 T. Magn Reson Med 2003; 49:576-580.

13. Kumar R, Delshad S, Woo MA, Macey PM, Harper RM. Age-related regional brain T2-relaxation changes in healthy adults. J Magn Reson Imaging 2012;35:300-308.

14. de Graaf RA, Brown PB, McIntyre S, Nixon TW, Behar KL, Rothman DL. High magnetic field water and metabolite proton T1 and T2 relaxation in rat brain in vivo. Magn Reson Med 2006;56:386-394.

15. Hu X, Norris DG. Advances in high-field magnetic resonance imaging. Annu Rev Biomed Eng 2004;6:157-184.

16. Budde J, Shajan G, Hoffmann J, Ugurbil K, Pohmann R. Human imaging at $9.4 \mathrm{~T}$ using $\mathrm{T}(2)^{*}$-, phase-, and susceptibility-weighted contrast. Magn Reson Med 2010;65:544-550.

17. Schepkin VD, Brey WW, Gor'kov PL, Grant SC. Initial in vivo rodent sodium and proton MR imaging at $21.1 \mathrm{~T}$. Magn Reson Imaging 2010;28:400-407.

18. van de Ven RC, Hogers B, van den Maagdenberg AM, de Groot HJ, Ferrari MD, Frants RR, Poelmann RE, van der Weerd L, Kiihne SR. $\mathrm{T}(1)$ relaxation in in vivo mouse brain at ultra-high field. Magn Reson Med 2007;58:390-395.

19. Kara F, van Dongen ES, Schliebs R, van Buchem MA, de Groot HJ, Alia A. Monitoring blood flow alterations in the Tg2576 mouse model of Alzheimer's disease by in vivo magnetic resonance angiography at 17.6T. NeuroImage 2012;60:958-966.
20. Kuo YT, Herlihy AH, So PW, Bhakoo KK, Bell JD. In vivo measurements of $\mathrm{T} 1$ relaxation times in mouse brain associated with different modes of systemic administration of manganese chloride. J Magn Reson Imaging 2005;21:334-339.

21. El Tannir El Tayara N, Delatour B, Le Cudennec C, Guegan M, Volk A, Dhenain M. Age-related evolution of amyloid burden, iron load, and MR relaxation times in a transgenic mouse model of Alzheimer's disease. Neurobiol Dis 2006;22:199-208.

22. Falangola MF, Dyakin VV, Lee SP, Bogart A, Babb JS, Duff K, Nixon R, Helpern JA. Quantitative MRI reveals aging-associated T2 changes in mouse models of Alzheimer's disease. NMR Biomed 2007;20: 343-351.

23. Weidensteiner C, Metzger F, Bruns A, Bohrmann B, Kuennecke B, von Kienlin M. Cortical hypoperfusion in the B6.PS2APP mouse model for Alzheimer's disease: comprehensive phenotyping of vascular and tissular parameters by MRI. Magn Reson Med 2009;62:35-45.

24. Waterston RH, Lindblad-Toh K, Birney E, et al., Mouse Genome Sequencing Consortium. Initial sequencing and comparative analysis of the mouse genome. Nature 2002;420:520-562.

25. Braakman N, Matysik J, van Duinen SG, Verbeek F, Schliebs R, de Groot HJ, Alia A. Longitudinal assessment of Alzheimer's beta-amyloid plaque development in transgenic mice monitored by in vivo magnetic resonance microimaging. J Magn Reson Imaging 2006;24: 530-536.

26. Carr HY, Purcell EM. Effects of diffusion on free precession in nuclear magnetic resonance experiments. Phys Rev 1954;94:630-638.

27. Meiboom S, Gill D. Modified spin-echo method for measuring nuclear relaxation times. Rev Sci Instrum 1958;29:688-691.

28. Hennig J, Nauerth A, Friedburg H. RARE imaging: a fast imaging method for clinical MR. Magn Reson Med 1986;3:823-833.

29. Corot C, Robert P, Idee JM, Port M. Recent advances in iron oxide nanocrystal technology for medical imaging. Adv Drug Deliv Rev 2006;58:1471-1504.

30. Rohrer M, Bauer H, Mintorovitch J, Requardt M, Weinmann HJ. Comparison of magnetic properties of MRI contrast media solutions at different magnetic field strengths. Invest Radiol 2005;40:715-724.

31. Muller R, Buttner P. A critical discussion of intraclass correlation coefficients. Stat Med 1994;13:2465-2476.

32. Bartzokis G, Mintz J, Marx P, Osborn D, Gutkind D, Chiang F, Phelan CK, Marder SR. Reliability of in vivo volume measures of hippocampus and other brain structures using MRI. Magn Reson Imaging 1993; 11:993-1006.

33. Bloembergen N, Purcell EM, Pound RV. Relaxation effects in nuclear magnetic resonance absorption. Phys Rev 1948;73:679-712.

34. Jezzard P, Duewell S, Balaban RS. MR relaxation times in human brain: measurement at $4 \mathrm{~T}$. Radiology 1996;199:773-779.

35. Klug G, Kampf T, Bloemer S, et al. Intracellular and extracellular T1 and $\mathrm{T} 2$ relaxivities of magneto-optical nanoparticles at experimental high fields. Magn Reson Med 2010;64:1607-1615.

36. Michaeli S, Garwood M, Zhu XH, DelaBarre L, Andersen P, Adriany G, Merkle H, Ugurbil K, Chen W. Proton T2 relaxation study of water, $\mathrm{N}$-acetylaspartate, and creatine in human brain using Hahn and Carr-Purcell spin echoes at 4T and 7T. Magn Reson Med 2002; 47:629-633.

37. Crawley AP, Henkelman RM. Errors in T2 estimation using multislice multiple-echo imaging. Magn Reson Med 1987;4:34-47.

38. Oh J, Han ET, Pelletier D, Nelson SJ. Measurement of in vivo multicomponent $\mathrm{T} 2$ relaxation times for brain tissue using multi-slice T2 prep at 1.5 and 3 T. Magn Reson Imaging 2006;24:33-43.

39. Majumdar S, Orphanoudakis SC, Gmitro A, O’Donnell M, Gore JC. Errors in the measurements of T2 using multiple-echo MRI techniques. II. Effects of static field inhomogeneity. Magn Reson Med 1986; 3:562-574.

40. Majumdar S, Orphanoudakis SC, Gmitro A, O’Donnell M, Gore JC. Errors in the measurements of T2 using multiple-echo MRI techniques. I. Effects of radiofrequency pulse imperfections. Magn Reson Med 1986;3:397-417.

41. Poon CS, Henkelman RM. Practical T2 quantitation for clinical applications. J Magn Reson Imaging 1992;2:541-553.

42. Whittall KP, MacKay AL, Graeb DA, Nugent RA, Li DK, Paty DW. In vivo measurement of T2 distributions and water contents in normal human brain. Magn Reson Med 1997;37:34-43.

43. Gareau PJ, Rutt BK, Bowen CV, Karlik SJ, Mitchell JR. In vivo measurements of multi-component T2 relaxation behaviour in guinea pig brain. Magn Reson Imaging 1999;17:1319-1325. 
44. Dydak U, Schar M. MR spectroscopy and spectroscopic imaging: comparing $3.0 \mathrm{~T}$ versus $1.5 \mathrm{~T}$. Neuroimaging Clin N Am 2006;16: 269-283.

45. Soher BJ, Dale BM, Merkle EM. A review of MR physics: 3T versus 1.5T. Magn Reson Imaging Clin N Am 2007;15:277-290.

46. Deelchand DK, Van de Moortele PF, Adriany G, Iltis I, Andersen P, Strupp JP, Vaughan JT, Ugurbil K, Henry PG. In vivo 1H NMR spectroscopy of the human brain at $9.4 \mathrm{~T}$ : initial results. J Magn Reson 2010;206:74-80.

47. Pohmann R, Shajan G, Balla DZ. Contrast at high field: relaxation times, magnetization transfer and phase in the rat brain at $16.4 \mathrm{~T}$. Magn Reson Med 2011;66:1572-1581.

48. Duewell SH, Ceckler TL, Ong K, Wen H, Jaffer FA, Chesnick SA, Balaban RS. Musculoskeletal MR imaging at $4 \mathrm{~T}$ and at $1.5 \mathrm{~T}$ : comparison of relaxation times and image contrast. Radiology 1995;196: 551-555.

49. Chen JH, Avram HE, Crooks LE, Arakawa M, Kaufman L, Brito AC. In vivo relaxation times and hydrogen density at $0.063-4.85 \mathrm{~T}$ in rats with implanted mammary adenocarcinomas. Radiology 1992;184: 427-434.

50. Cremillieux Y, Ding SJ, Dunn JF. High-resolution in vivo measurements of transverse relaxation times in rats at 7 Tesla. Magn Reson Med 1998;39:285-290.

51. Fischer HW, Rinck PA, Van Haverbeke Y, Muller RN. Nuclear relaxation of human brain gray and white matter: analysis of field dependence and implications for MRI. Magn Reson Med 1990;16: 317-334.

52. Bartha R, Michaeli S, Merkle H, Adriany G, Andersen P, Chen W, Ugurbil K, Garwood M. In vivo ${ }^{1} \mathrm{H}_{2} \mathrm{O}$ T2* measurement in the human occipital lobe at $4 \mathrm{~T}$ and $7 \mathrm{~T}$ by Carr-Purcell MRI: detection of microscopic susceptibility contrast. Magn Reson Med 2002;47:742-750.

53. Hardy PA, Henkelman RM. Transverse relaxation rate enhancement caused by magnetic particulates. Magn Reson Imaging 1989;7: $265-275$.
54. Brooks RA, Vymazal J, Bulte JWM, Baumgarner CD, Tran V. Comparison of T2 relaxation in blood, brain, and ferritin. J Magn Reson Imaging 1995;5:446-450.

55. Stefanovic B, Sled JG, Pike GB. Quantitative T-2 in the occipital lobe: the role of the CPMG refocusing rate. J Magn Reson Imaging 2003;18:302-309.

56. Jackson GD, Connelly A, Duncan JS, Grunewald RA, Gadian DG. Detection of hippocampal pathology in intractable partial epilepsy: increased sensitivity with quantitative magnetic resonance T2 relaxometry. Neurology 1993;43:1793-1799.

57. Briellmann RS, Kalnins RM, Berkovic SF, Jackson GD. Hippocampal pathology in refractory temporal lobe epilepsy: T2-weighted signal change reflects dentate gliosis. Neurology 2002;58:265-271.

58. Chen JC, Hardy PA, Clauberg M, Joshi JG, Parravano J, Deck JH, Henkelman RM, Becker LE, Kucharczyk W. T2 values in the human brain: comparison with quantitative assays of iron and ferritin. Radiology 1989;173:521-526.

59. Santyr GE. Magnetization transfer effects in multislice MR imaging. Magn Reson Imaging 1993;11:521-532.

60. Watanabe A, Boesch C, Obata T, Anderson SE. Effect of multislice acquisition on $\mathrm{T} 1$ and $\mathrm{T} 2$ measurements of articular cartilage at 3T. J Magn Reson Imaging 2007;26:109-117.

61. Saito N, Sakai O, Ozonoff A, Jara H. Relaxo-volumetric multispectral quantitative magnetic resonance imaging of the brain over the human lifespan: global and regional aging patterns. Magn Reson Imaging 2009;27:895-906.

62. Oros-Peusquens AM, Laurila M, Shah NJ. Magnetic field dependence of the distribution of NMR relaxation times in the living human brain. Magn Reson Mater Phys 2008;21:131-147.

63. Cho S, Jones D, Reddick WE, Ogg RJ, Steen RG. Establishing norms for age-related changes in proton T-1 of human brain tissue in vivo. Magn Reson Imaging 1997;15:1133-1143.

64. Steen RG, Gronemeyer SA, Taylor JS. Age-related changes in proton T1 values of normal human brain. J Magn Reson Imaging 1995;5:43-48. 